\title{
Allelopathic Effects of Nicotiana tabacum on the Germination of Vigna radiata and Triticum aestivum
}

\author{
Jason M. Baek ${ }^{1}$, Olivia J. Kawecki ${ }^{1}$, Krishawn D. Lubin ${ }^{1}$, Jialin Song ${ }^{1}$, Olivia A. \\ Wiens ${ }^{1}$, Fiona $\mathrm{Wu}^{1}$ \\ ${ }^{1}$ University of Western Ontario, Department of Biology
}

\begin{abstract}
Allelopathic chemicals in many plants can be released into the surrounding soil and may affect the development of nearby crops. Understanding allelopathic effects can be extremely beneficial for both economic and environmental reasons. The information gained from understanding allelopathy may be used in the optimization of crop rotations. Previous studies have investigated the negative allelopathic effects of Nicotiana tabacum - tobacco, on corn and other crops. Our study investigated the allelopathic impact of tobacco on seed germination of mung bean, Vigna radiata, and organic red fife wheat, Triticum aestivum. Seeds were treated with various concentrations of tobacco leaf solution to study the effects of tobacco on germination of the seeds.

The effects of tobacco on the germination rate and percentage of germination were analyzed using one-way ANOVA with Tukey HSD tests. Results showed that there was a significant decrease in germination rate at high concentrations of tobacco. Our findings suggest that allelopathic chemicals released by tobacco have detrimental effects on the germination of mung bean and red fife wheat. Therefore, when forming crop rotations, it is important to take into account and understand the allelopathic effects and interactions between different species planted in the same region.
\end{abstract}

\section{Introduction}

Growth, survival, and reproduction of plants can be influenced by biochemicals produced by surrounding vegetation; this phenomenon is known as allelopathy. These secreted chemicals may be beneficial because they can ensure a plant's longterm survival against competing plants (1). There are a myriad of effects associated with allelochemicals. They can inhibit the following: plant growth, the uptake of nutrients, or they may impede on the plant's ability to utilize acquired resources (2).

The precise biological mechanisms of allelopathic effects require further research. Not all plants induce allelopathic effects on their surrounding plant communities. Many plants utilize alternative physical methods to compete with nearby plants (i.e. larger leaf size to compete for sunlight) (1). It can be difficult to determine whether plants compete by chemical or physical means. Moreover, there can be beneficial (positive allelopathy) or detrimental (negative allelopathy) effects on a target organism (2).

In agricultural settings where crop rotation is often implemented, allelopathic competition may reduce crop yields (3). Crop rotation is a beneficial practice where a particular crop is grown in a specific region, and a different crop is grown in the same region afterwards (4). By changing the crops grown in the same location on a seasonal basis, the soil is less prone to nutrient loss by the activity of one specific crop, thus increasing the presence of various nutrients in the soil and ultimately increasing soil fertility as well as crop yield (4). However, the soil may also contain remaining allelochemicals left over from the previous crop, and these residual effects may influence the growth of subsequent crops grown (5).

The objective of this experiment was to investigate the allelopathic effects of tobacco, Nicotiana tabacum (L.) on mung bean, Vigna radiata ((L.) R. Wilczek) and on organic red fife wheat, Triticum aestivum (L.). These species were chosen because both mung bean and red fife wheat are important cultivated plants widely grown for food around the world. Growing tobacco, which has known allelopathic effects on other plants such as maize may stunt the growth of mung bean and red fife wheat when practicing crop rotation (5).

Previous studies have found that allelopathic chemicals from tobacco negatively impacts mung bean growth (5). However, their research focuses on measuring various factors including number of leaves per plant, plant height, seedling dry weight, and enzymatic activity, but does not specifically address the length of the radicle during germination (5). Moreover, research has yet to be conducted on the allelopathic effects of tobacco on red fife wheat. Red fife wheat is grown in over 150 countries and is considered one of the most commonly cultivated wheats in the world (6). Thus, it is necessary to know whether there are carry-over effects of planting 
different crops, such as red fife wheat, in the same field where tobacco has been grown.

To study these possible effects, the germination-emergence of a radicle-of mung bean and red fife wheat was studied. Germination is a relatively simple method to study plant growth, since the length of the radicle can be visibly seen, measured and compared under different growth conditions. Germination is also a good indicator of plant health and viability since seeds will not germinate if there are unsuitable or extreme environmental conditions (i.e. low or high temperature) (7). Furthermore, because germination is the first visible stage of plant growth, other ways of measuring plant growth (i.e. shoot length, root:shoot ratio) were rejected for timing reasons, ambiguity in distinguishing plant parts, and potential influences of other unforeseen factors later in the growth process. Previous research done on the effects of allelochemicals on germination show that allelochemicals like abscisic acid and ephedrine have negative effects on Coronilla varia (crownvetch) seeds by increasing germination time and decreasing germination rate, therefore the effects of allelochemicals on germination was a suitable topic to study (8).

It was hypothesized that tobacco would have allelopathic effects on the germination of mung bean and organic red fife wheat. It was predicted that both the rate of germination and percentage germination of mung bean and organic red fife wheat would decrease when exposed to increasing concentrations of tobacco leaf solution.

\section{Methods}

The experiment tested effects of tobacco leaf solution at various concentrations on the germination of mung beans and organic red fife wheat seeds. There were five treatments of tobacco leaf solution of $0 \%$ (control treatment), $3 \%, 8 \%, 13 \%$, and $18 \%$. The tobacco leaf solution was prepared by using $100.0 \mathrm{~g}$ of dried and crushed tobacco leaves and $750.0 \mathrm{~mL}$ distilled water, which was then placed in a growth chamber for 48 hours at $25.0^{\circ} \mathrm{C}$. A cheese cloth was used to filter the leaves from the solution. The tobacco solution was then diluted using distilled water to the appropriate concentrations used in this experiment. For each treatment, 20 seeds of each species were randomly selected, placed in a Petri dish and subjected to $6.0 \mathrm{~mL}$ of one of the treatment solutions. Each treatment was replicated six times for both test organisms. There was a total of 60 Petri dishes, each containing 20 seeds. Germination of the seeds was monitored daily for five days to collect data, and the criterion for germination was a $2.0 \mathrm{~mm}$ radicle length. During this time, the Petri dishes were placed in a growth chamber at $25.0^{\circ} \mathrm{C}$ alternating between 14 hours in light and 10 hours in darkness. If any of the plates dried out, they were replenished with 1.0-2.0 mL of treatment solution. The collected data were placed into IBM SPSS Statistics 22 (2014) and analyzed using one-way ANOVA and Tukey tests.

\section{Results}
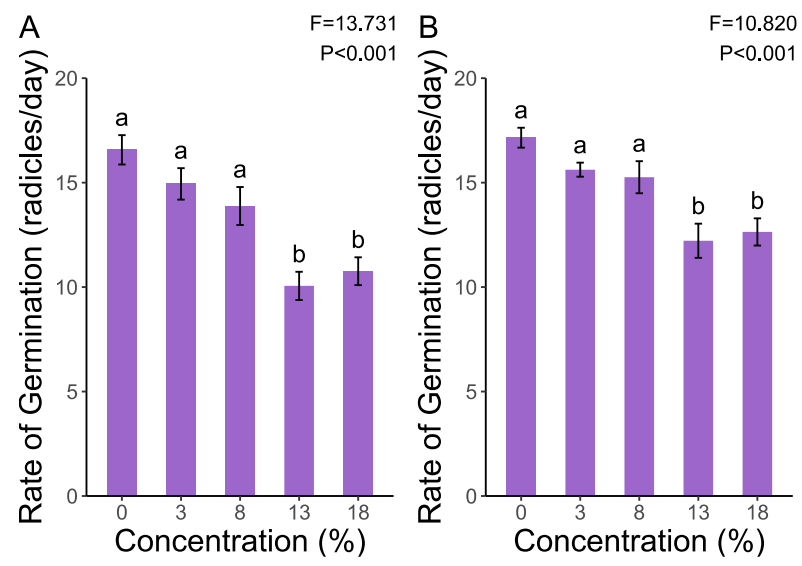

Fig. 1. Effect of various tobacco leaf solution concentrations (\%) on the mean \pm SE of rate of germination ( $2 \mathrm{~mm}$ radicles/day) of plant species red fife wheat $(A)$ and mung bean (B). Means followed by the same letter within each variable are not significantly different $(P<0.05)$ according to Tukey's HSD test.

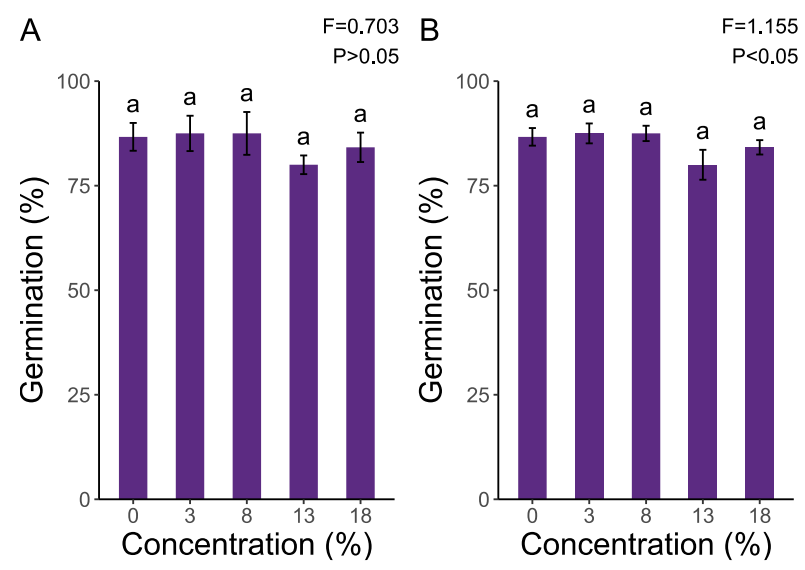

Fig. 2. Effect of various tobacco leaf solution concentrations (\%) on the mean \pm SE of percentage germination (\%) of plant species red fife wheat $(A)$ and mung bean (B). Means followed by the same letter within each variable are not significantly different $(P<0.05)$ according to Tukey's HSD test.

The rate of germination for red fife wheat after being subjected to $0 \%$ tobacco leaf solution was significantly higher $(F=13.73, P<0.001)$ than the rate 
of germination after exposure to $13 \%$ and $1 \%$ tobacco leaf solutions (Fig. 1. A.). Similarly, the rate of germination for mung bean was significantly higher $(F=10.82, P<0.001)$ for seeds exposed to $0 \%$ tobacco leaf solution compared to concentrations of $13 \%$ and $18 \%$. The effect of tobacco concentrations at $0 \%, 3 \%$ and $8 \%$ had no significant differences between them, and led to higher rates of germination for both plant species. There were no significant differences between concentrations at $13 \%$ and $18 \%$ on the rate of germination in both red fife wheat and mung bean (Fig. 1).

Various concentrations of tobacco leaf solution had no significant effects on the percentage germination of red fife wheat and mung bean (Fig. 2). Seeds of both plant species germinated to a similar extent for all concentrations. Higher tobacco concentrations at $13 \%$ and $18 \%$ show slight decrease in percentage germination; however, the difference is not significant (Fig. 2).

\section{Discussion}

In this experiment, it was hypothesized that tobacco leaves would have negative allelopathic effects on red fife wheat and mung bean. The results did match initial predictions for germination rate. When the two species were exposed to increasing concentrations of tobacco leaf solution, the resulting percent germination had no significant differences; whereas, the germination rate decreased significantly when exposed to the same conditions. In our experiment, tobacco concentrations below $20 \%$ were used, which are high enough to observe its allelopathic effects on seeds, but not high enough to prevent seed germination altogether, which may explain why there were no significant differences in percent germination.

The tobacco treatment solution was prepared by soaking crushed tobacco leaves in distilled water, then discarding the leaves. This method of filtering out the allelochemicals resembles the allelopathic nature of plants in soil, since many allelochemicals are leached out of living plants or plant residue by rain or dew (9). It is more realistic to treat the target plants with an aqueous solution in comparison to placing dry tobacco leaves in close proximity or in contact with the target plants. Moreover, the target plants often take up nutrients and allelochemicals by absorption in the roots which further supports the use of an aqueous solution.

Crushing the leaves increases their surface area, allowing a greater amount of allelochemicals to be released into solution. The nicotine concentration in tobacco leaves is only $0.1-1 \%$ of the tobacco plant's dry mass, so it was important to extract as many of the allelochemicals as possible from the leaves (10). Nicotine is synthesized in the roots of tobacco plants as a defence mechanism to physical damage, so in the fields, the mung beans and red fife wheat seeds may be exposed to higher concentrations of tobacco allelochemicals than the concentrations used in this experiment (11). This is because any physical damage to the living tobacco plants will result in higher concentrations of nicotine production in the roots and therefore a higher concentration of nicotine will be leached out into the soil and consequently taken up by the target plants.

The allelochemical concentrations used were not high enough to demonstrate a significant decrease in percent germination, however the significant decrease in germination rate as well as the potential for higher allelochemical concentrations in the soil highlights the possibility that in real practice, percent germination may significantly decrease. Adding more of the treatment solution to dried dishes did increase the amount of allelochemicals in each dish, however, the initial tobacco concentrations were fairly low so any additional drops only resulted in a minor increase of allelochemicals.

Tobacco had negative effects on the mung bean and red fife wheat seeds by decreasing the germination rate. Tobacco increases the presence of secondary metabolites such as alkaloids, all of which may have different effects on mung bean as well as red fife wheat seed growth. One predominant alkaloid is nicotine, which significantly decreases growth uniformity $(12,13)$. It is possible that after filtration, the nicotine in the tobacco leaf solution was absorbed by the two seed species. Negative effects of nicotine on humans is a widely researched topic, but despite this knowledge base, there still remains insufficient information of these effects on plants.

Another possible explanation for the decreased germination rate relates back to recent findings showing that several allelopathic compounds are structurally similar to plant hormones (13). Some mechanisms of action for certain allelochemicals resemble those of plant hormones such as gibberellin - a hormone produced in the stem and roots of plants that helps regulate stem elongation (14). These released allelochemicals may have inhibited gibberellin and disrupted enzymes required for germination, thus inhibiting the germination rate of the mung beans and red fife wheat seeds.

Growing seasons are finite in length and if crops are not grown within this period of time, weather or other environmental effects caused by the changing of seasons could destroy crops and/or 
reduce yield. Allelochemicals can negatively affect the length of this period of optimal growth, and thus, understanding the allelopathic relationships between crops within a rotation may prevent yield degradation and provide amplified economic benefits. In order to maximize crop yield, it is beneficial to research the impact of growing tobacco with organic red fife wheat and mung beans when following the practice of crop rotation.

Along with crop rotation, intercropping is another practice where two or more crops are grown in the same region at the same time in a specific row arrangement (15). If tobacco and either mung bean or red fife wheat are grown together using this practice, it would be important to understand the allelochemical effects that could occur. Intercropping presents another concern due to the close proximity of the plants. Absorption through the roots is not the only way to be exposed to allelochemicals; other practical ways of spreading allelochemicals includes root grafting (roots joining and growing together) and common fungi (fungi being shared by the roots of different plants) (9). However, this experiment only addresses the possible allelopathic effects of tobacco on mung bean and red fife wheat, and not the effects of mung bean and red fife wheat on tobacco or on each other.

In addition, tobacco contains chemicals that are used in botanical insecticides - most notably nicotine (16). Therefore, if one wanted to use a pesticide on crops, it would be important to know not only whether an ingredient could kill the pest, but also to know if it contains allelochemicals that could induce changes in the crop yield. Further research is also required to investigate the nature of the biological mechanism of alkaloids, specifically nicotine, on plants. For future experiments, the time taken for germination to occur could be measured to see if higher tobacco concentrations results in longer germination times. More research could also be done to see if there is a concentration limit for tobacco allelochemicals in which significant differences in percent germination exist. This would also be beneficial for the application of pesticides containing nicotine since pesticide concentrations could be established in which no significant harmful effects on crop yield would arise.

More insight into the long-term allelopathic effects of tobacco could be gained if the experiment was carried out for an extended period of time. In this way, more factors pertaining to both the target plants and the allelochemicals could be studied. In the case of the target plants, plant growth could be measured in other ways (i.e. weight, plant height, number of leaves, surface area of leaves, observations on plant colour and form, biochemical measurements) to provide a more thorough understanding of allelopathic effects. In the case of the allelochemicals, nutrients and organic compounds in the nearby soil could be measured and studied to examine if the presence of tobacco leads to the leaching or addition of certain nutrients in the soil. These compounds may then influence the growth of plants cultivated in the same region afterwards.

It is also important to examine beyond allelopathic effects on the target plants by considering the long-term allelochemicals that will be present in the soil, long after the tobacco plant is removed (17). However, there is also the likelihood that many of these allelochemicals will either deteriorate or be leached from the soil over time. Although this experiment was not performed in the field, previous research pursuing a crop rotation design with seasonal crop changes shows the negative allelochemical effects of tobacco on mung bean in the field (5). Therefore, there is still a significant presence of allelochemicals in the soil that do not decay or disappear in a season. Overall, even if this may not serve as a present concern for the growth of target plants, these persistent allelochemicals will have greater importance in the ecosystem as they continue to influence the growth of nearby species (17).

\section{Conclusion}

There was an observed allelopathic effect of tobacco on the germination of mung bean and organic red fife wheat. Although there was no significant increase or decrease in the percentage of germinated seeds, the germination rate significantly decreased as the concentration of tobacco leaf solution increased to $13 \%$ and $18 \%$.

\section{References}

1. Venturelli $S$, et. al. Plants release precursors of histone deacetylase inhibitors to suppress growth of competitors. Plant Cell. 2015; 27(11):3175-3189. DOI: 10.1105/tpc.15.00585

2. Batish DR, Singh HP, Kaur S, Kohli RK and Yadav SS. Caffeic acid affects early growth, and morphogenetic response of hypocotyl cuttings of mung bean (Phaseolus aureus). J Plant Physiol. 2008; 165: 297-305. DOI: 10.1016/j.jplph.2007.05.003

3. Wu H, Pratley J, Lemerle D and Haig T. Allelopathy in wheat (Tritcum aestivum). Ann Appl Biol. 2001; 139 (1): 1-9. DOI:10.1111/j.1744-7348.2001.tb00124.x

4. Marais A, Hardy M, Booyse $M$ and Botha A. Effects of Monoculture, Crop Rotation, and Soil Moisture Content on Selected Soil Physicochemical and Microbial Parameters in Wheat Fields. Appl Environ Soil Sci. 2012; 12:1-13. DOI:10.1155/2012/593623

5. Farooq M, Hussain T, Wakeel A and Cheema ZA. Differential response of maize and mungbean to tobacco allelopathy. Expl Agric. 2014; $50 \quad$ (4): 611-624. DOI: $10.1017 / \mathrm{S} 0014479714000106$ 
6. Mason, H. E. and Spaner, D. Competitive ability of wheat in conventional and organic management systems: A review of the literature. Can. J. Plant Sci. 2006; 86(2): 333-343. DOI: 10.4141/P05-051

7. Center for Environmental Farming Systems. Crop Rotations on Organic Farms. North Carolina: North Carolina Cooperative Extension Service; 2006.

8. Isfahan MN and Shariati M. The Effect of Some Allelochemicals on Seed Germination of Coronolla varia L. Seeds. AmericanEurasian J. Agric. \& Environ. Sci. 2007; 2 (5): 534-538.

9. Rice EL. Allelopathy. 2nd ed. Florida: Academic Press; c1984. p. 310-319.

10. Xi X, Li C and Zhang F. Tobacco plants can use nitrogen taken up before mechanical wounding to synthesize nicotine afterwards. Plant Signal Behav. 2008; 3(2): 87-90. DOI: 10.4161/psb.3.2.5121

11. Tso TC and Jeffrey RN. Studied on tobacco alkaloids. II. The formation of nicotine and nornicotine in tobacco supplied with N15. Plant Physiol. 1957; 32(2):86-92.

12. Baldwin, IT. Mechanism of damage-induced alkaloid production in wild tobacco. J Chem Ecol. 1989; 15 (5): 1661 1680. DOI: $10.1007 / \mathrm{BF} 01012392$
13. Kruse, M., M. Strandberg and B. Strandberg. 2000. Ecological effects of allelopathic plants - A Review. Ministry of Environment and Energy, National Environmental Research Institute, NERI Technical Report, No. 315, Silkeborg, Denmark, pp: 67.

14. Wink M, Schmeller T and Latz-Bruning B. Modes of action of allelochemical alkaloids: Interaction with neuroreceptors, DNA, and other molecular targets. J Chem Ecol. 1998; 24 (11): 1881-1937. DOI: 10.1023/A:1022315802264

15. Vandermeer JH. The Ecology of Intercropping. New York: Cambridge University Press; c1989. p. 6.

16. Casanova H, Ortiz C, Peláez C, Vallejo A, Moreno ME and Acevedo M. Insecticide Formulations Based on Nicotine Oleate Stabilized by Sodium Caseinate. J. Agric. Food Chem. 2002; 50(22): 6389-6394. DOI: 10.1021/jf0257244

17. Del Fabbro $C$ and Prati D. The relative importance of immediate allelopathy and allelopathic legacy in invasive plant species. Basic Appl Ecol. 2014; 16(1): 28. DOI: 10.1016/j.baae.2014.10.007 